Article

\title{
Conventional Study on Novel Dicationic Ionic Liquid Inclusion with $\beta$-Cyclodextrin
}

Sharifah Mohamad ${ }^{1, *}$, Hemavathy Surikumaran ${ }^{1}$, Muggundha Raoov ${ }^{1,2}$, Tilagam Marimuthu ${ }^{1}$, Kumuthini Chandrasekaram ${ }^{1}$ and Puvaneswary Subramaniam ${ }^{1}$

1 University of Malaya Centre for Ionic Liquids, Department of Chemistry, Faculty of Science, University of Malaya, Kuala Lumpur 50603, Malaysia; E-Mails: hems8620@yahoo.com (H.S.); vainavi38@gmail.com (T.M.); kumuthinic@um.edu.my (K.C.); puva@um.edu.my (P.S.)

2 Advanced Medical \& Dental Institute, University of Science Malaysia, No 1-8 (Lot 8), Persiaran Seksyen 4/1, Bandar Putra Bertam, Kepala Batas, Pulau Pinang 13200, Malaysia; E-Mail: muggundha_raoov@hotmail.com

* Author to whom correspondence should be addressed; E-Mail: sharifahm@um.edu.my; Tel.: +603-79676751; Fax: +603-79674193.

Received: 27 June 2011; in revised form: 25 August 2011 / Accepted: 25 August 2011 / Published: 23 September 2011

Abstract: This study focuses on the synthesis and characterization of the inclusion complex of $\beta$-Cyclodextrin ( $\beta$-CD) with dicationic ionic liquid, 3,3'-(1,4-Phenylenebis [methylene]) bis(1-methyl-1H-imidazol-3-ium) di(bromide) (PhenmimBr). The inclusion complex was prepared at room temperature utilizing conventional kneading technique. Proton $\left({ }^{1} \mathrm{H}\right)$ NMR and 2D $\left({ }^{1} \mathrm{H}-{ }^{1} \mathrm{H}\right)$ COSY NMR were the primary characterization tools employed to verify the formation of the inclusion complex. COSY spectra showed strong correlations between protons of imidazolium and protons of $\beta-\mathrm{CD}$ which indicates that the imidazolium ring of PhenmimBr has entered the cavity of $\beta$-CD. UV absorption indicated that $\beta$-CD reacts with PhenmimBr to form a $2: 1 \beta$-CD-PhenmimBr complex with an apparent formation constant of $2.61 \times 10^{5} \mathrm{~mol}^{-2} \mathrm{~L}^{2}$. Other characterization studies such as UV, FT-IR, XRD, TGA, DSC and SEM studies were also used to further support the formation of the $\beta$-CD-PhenmimBr inclusion complex.

Keywords: dicationic ionic liquid; cyclodextrin; inclusion complex 


\section{Introduction}

Supramolecular chemistry is a discipline of chemistry which has been attracting much attention recently, especially the host-guest type interaction. Among all the potential hosts, Cyclodextrin (CD) is the most significant one [1]. CDs are well known series of macro cyclic oligosaccharides resulting from the degradation of starch by bacterial enzymes [2]. Generally, CDs are composed of 6, 7, or 8 glucose units connected by $\alpha-1,4-$ glucosidic linkages which are categorized as $\alpha-\beta-$ and $\gamma-C D$, respectively [3-6]. Among these CDs, $\beta$-Cyclodextrin $(\beta-C D)$ has been chosen for this study since its cavity size is suitable for guest molecules with molecular weights between 200 and $800 \mathrm{~g} / \mathrm{mol}[7,8]$. $\beta-C D$ can accommodate variety of organic and inorganic compounds to form inclusion complexes (ICs) due to its hydrophobic cavity [9,10] thus it is being widely used in pharmaceutical industry [11], foodstuff [12,13], separation studies [14] and environmental engineering [15,16].

An ionic liquids (IL), is a salt in which the ions are poorly coordinated. Consequently these compounds are liquid below $100{ }^{\circ} \mathrm{C}$ or even at room temperature (RTIL's). They remain in the liquid state over a wide temperature range, and have unique properties such as non-volatility, non-flammability, low viscosity, chemical and electrochemical stability [17]. Recently, ionic liquid is being considered as a new and remarkable class of solvents as they are good solvents for a wide range of organic and inorganic materials [18]. According to Wilkes the recent explosion of interest in the ILs [19] is due to the realization that these materials, used mostly for specialized electrochemical applications may have greater utility as reaction solvents. In comparison with monocationic ILs, multifunctional ILs (dicationic and dianionic ILs) have potential to exhibit a greater range of physical properties [20] in terms of greater thermal stability, lower volatility, and more flexibility in tuning/varying their physicochemical properties.

Understanding the interaction between ILs and CDs is important to analytical chemistry and material synthesis [21-24]. It was found that ILs could solubilize a number of complex organic molecules such as CDs which is very useful in separation community. Due to high solubility with CDs, ILs has been providing additional selectivity and resolution for separations. Successful separation with the combination of CDs and ILs suggests that there may be interactions between CDs and ILs. Therefore study of inclusion complex between CDs and ILs is very important to investigate the actual nature of the interaction. There are several reports on CDs with monocationic ILs [8,10,25,26-29]. To the best of our knowledge, studies on the inclusion complexation of dicationic IL with $\beta$-CD in the scientific literature are scarce [30]. Hence in this study, the binding nature of the dicationic IL (PhenmimBr) with $\beta-\mathrm{CD}$ is analysed and compared with monocationic ILs.

\section{Experimental}

\subsection{Reagent and Solution}

$\beta-\mathrm{CD}$ is commercially available and was purchased from Acros (Hungary) (99\%). $\alpha, \alpha$-dibromo- $p$-xylene and 1-methylimidazole was supplied by Merck. Other reagents and chemicals were of analytical reagent grade and were used as received without further purification. Double distilled water was used throughout the experiment. 


\subsection{Instrumentation}

The Proton Nuclear Magnetic Resonance ${ }^{1} \mathrm{H}$ (NMR) and Correlation Spectroscopy (COSY) spectra of the samples in dimethyl sulfoxide (DMSO) were recorded on Lambda JEOL $400 \mathrm{MHz}$ Fourier Transform NMR (FT-NMR) spectrometer at room temperature. Calibration of proton chemical shift was achieved by using tetramethylsilane (TMS) as an internal reference standard. The Fourier Transform Infrared (FT-IR) spectra were recorded on a Perkin-Elmer RX1 FT-IR spectrometer with samples prepared using potassium bromide $(\mathrm{KBr})$ pellets. All the samples were run in the spectral region range of 400-4000 $\mathrm{cm}^{-1}$. X-ray diffraction (XRD) patterns were taken using $\mathrm{Cu} \mathrm{K} \alpha$ irradiation with a Siemens D5000 X-ray diffractometer (voltage, $40 \mathrm{kV}$; current, $100 \mathrm{~mA}$ ). Powder samples were mounted on a sample holder and scanned from $5^{\circ}$ to $30^{\circ}$ at a speed of $3^{\circ}$ per minute. Thermo gravimetric analyses (TGA) curves were examined using a TA Instruments Q500. A linear heating rate was set at $20^{\circ} \mathrm{C}$ per minute within the temperature range from $50{ }^{\circ} \mathrm{C}$ to $900{ }^{\circ} \mathrm{C}$ in a stream of nitrogen atmosphere. Differential Scanning Calorimetry (DSC) analysis was done by heating the samples from $36{ }^{\circ} \mathrm{C}$ to $350{ }^{\circ} \mathrm{C}$ at $5{ }^{\circ} \mathrm{C}$ per minute. Spectrophotometric measurements were made with a Shimadzu Ultraviolet-Visible spectroscopy (UV-Vis) recording spectrophotometer equipped with $1 \mathrm{~cm}$ quartz cells.

\subsection{Synthesis}

2.3.1. Preparation of 3,3'-(1,4-Phenylenebis [methylene]) bis(1-methyl-1H-imidazol-3-ium) di(bromide) (PhenmimBr)

PhenmimBr was prepared as reported previously by Kilivelu and Yatimah [31]. The mixture of $\alpha, \alpha$-dibromo- $p$-xylene and 1-methylimidazole (1:2) were dissolved in acetonitrile and the solution refluxed at $60{ }^{\circ} \mathrm{C}$. The reaction product was allowed to cool to room temperature and deposit overnight. The resulting white precipitate was collected by vacuum filtration, washed with acetone several times and dried in a vacuum oven. The end product obtained was a white powder with the calculated yield of $72 \%$. The reaction mechanism for the synthesis of PhenmimBr from the starting materials, 1-methylimidazole and $\alpha, \alpha$-dibromo- $p$-xylene is shown in Figure 1. The reaction mechanism shows that the PhenmimBr is produced by a simple aromatic nucleophilic substitution reaction.

Figure 1. Synthesis of PhenmimBr.

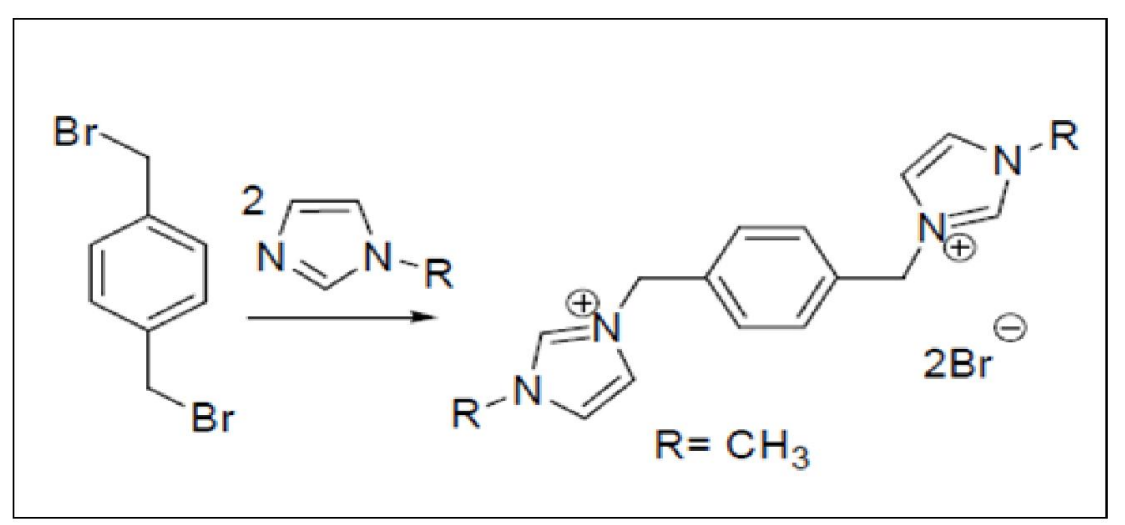




\subsubsection{Synthesis of $\beta$-Cyclodextrin-PhenmimBr ( $\beta$-CD-PhenmimBr)}

The inclusion complex of $\beta-\mathrm{CD}$ with PhenmimBr was prepared using conventional kneading method [32]. Equimolar amounts of $\beta-\mathrm{CD}$ and PhenmimBr was kneaded with mortar and pestle in minimal ethanol to form a homogeneous paste. The complex was kneaded for approximately 30 minutes and dried to constant mass. After drying, a white powder ( $\beta$-CD-PhenmimBr complex) was obtained. The calculated yield was $78 \%$.

\subsection{Procedure}

\subsubsection{Preparation of $\beta$-CD-PhenmimBr for Spectroscopic Studies}

A $2.0 \mathrm{~mL}$ portion of $0.01 \mathrm{mM}$ PhenmimBr aliquot and $3.2 \mathrm{~mL}$ of $0.0032 \mathrm{M} \beta$-CD solution was adjusted to $\mathrm{pH} 7.0$, transferred accurately into a $10.0 \mathrm{~mL}$ standard volumetric flask and diluted to the mark with double distilled water. The absorption spectrum of $\beta$-CD-PhenmimBr complex was recorded against blank reagent which was prepared with the same reagent concentration but without the addition of PhenmimBr. In addition, absorption spectra of PhenmimBr and $\beta-\mathrm{CD}$ were also recorded. All the absorbance was measured at $200 \mathrm{~nm}$ separately against blank reagent. For the formation constant curve, the concentration of PhenmimBr was held constant at $0.01 \mathrm{mM}$, meanwhile the concentration of $\beta$-CD was varied $(0,0.001,0.002,0.003$ and $0.004 \mathrm{M})$. This procedure was replicated in order to obtain three or more absorbance values for each of the $\beta-C D$ concentration studied.

\section{Results and Discussion}

\section{1. ${ }^{1}$ H Nuclear Magnetic Resonance (NMR) Spectra}

${ }^{1} \mathrm{H}$ NMR spectra is a useful technique to confirm the formation of an inclusion complex and can provide useful information on the inclusion mechanism of CDs with the guest molecules. Chemical shift changes of specific nuclei in the host molecule can verify the formation of inclusion complex in solution, since significant changes in microenvironment are known to occur in $\mathrm{CD}$ of the inclusion complex [32]. The obvious upfield shift of the protons on the inner cavity of $\beta-\mathrm{CD}$, i.e., $\mathrm{H} 3$ and $\mathrm{H} 5$ were observed due to anisotropic shielding by ring current from the aromatic rings of PhenmimBr (Table 1). The H1, H2 and $\mathrm{H} 4$ protons of $\beta-\mathrm{CD}$, on the outer part of the cavity (Figure 2) also faces an upfield shift but do not exhibit considerable changes upon addition of PhenmimBr. The same phenomenon is observed with the H6 proton. From the ${ }^{1} \mathrm{H}$ NMR data, it can also be surmised that when PhenmimBr enters the hydrophobic cavity of $\beta-C D$, the change of the micro-environment in PhenmimBr protons lead to the upfield shift of the protons. $\mathrm{Hb}$ and $\mathrm{Hd}$ protons of the imidazolium ring (Figure 3) shows obvious shift upon inclusion complex formation. The presence of ${ }^{1} \mathrm{H}$ signals belonging to both $\beta-\mathrm{CD}$ and PhenmimBr molecules could be observed in ${ }^{1} \mathrm{H}$ NMR spectrum of $\beta$-CD-PhenmimBr which strongly suggests that the new inclusion complex has been formed. 
Table 1. Chemical shifts $(\delta)$ of $\beta-C D$, PhenmimBr and $\beta-C D-P h e n m i m B r$.

\begin{tabular}{ccccc}
\hline & $\boldsymbol{\beta}-\mathbf{C D}$ & PhenmimBr & $\boldsymbol{\beta}$-CD-PhenmimBr & \\
\hline & $\boldsymbol{\delta}$ & $\boldsymbol{\delta}$ & $\boldsymbol{\delta}$ & $\Delta \boldsymbol{\delta}$ \\
\hline $\mathrm{H} 1$ & 4.8191 & & 4.8094 & -0.0097 \\
$\mathrm{H} 2$ & 3.3144 & & 3.2955 & -0.0189 \\
$\mathrm{H} 3$ & 3.6402 & & 3.5987 & $-\mathbf{0 . 0 4 1 5}$ \\
H4 & 3.3607 & & 3.3504 & -0.0103 \\
H5 & 3.6006 & & 3.5591 & $-\mathbf{0 . 0 4 1 5}$ \\
H6 & 3.6469 & & 3.6189 & -0.0280 \\
\hline Ha & & 3.8532 & 3.8410 & -0.0122 \\
Hb & & 9.3449 & 9.2430 & $-\mathbf{0 . 1 0 1 9}$ \\
Hc & & 5.4598 & 5.4226 & -0.0372 \\
Hd & & 7.8243 & 7.7633 & $-\mathbf{0 . 0 6 1 0}$ \\
He & & 7.7291 & 7.6974 & -0.0317 \\
Hf & & 7.4948 & 7.4637 & -0.0311 \\
\hline
\end{tabular}

Figure 2. Assignation of protons in $\beta-C D$.

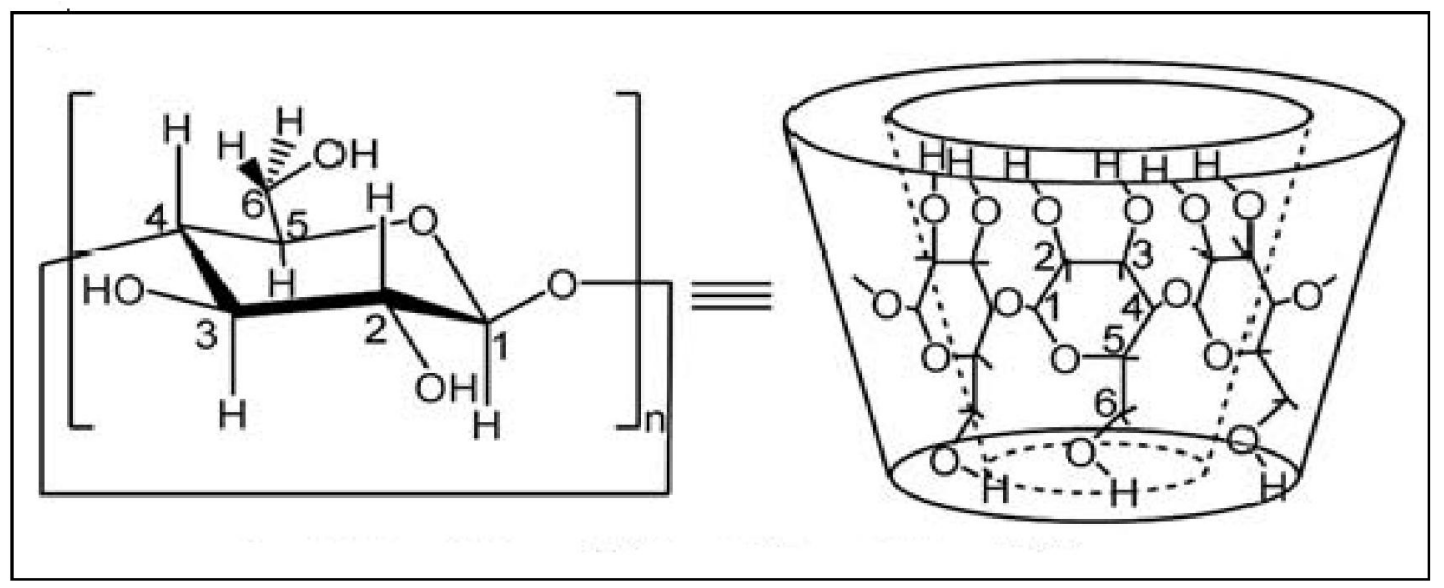

Figure 3. Structure of PhenmimBr with labeled protons.

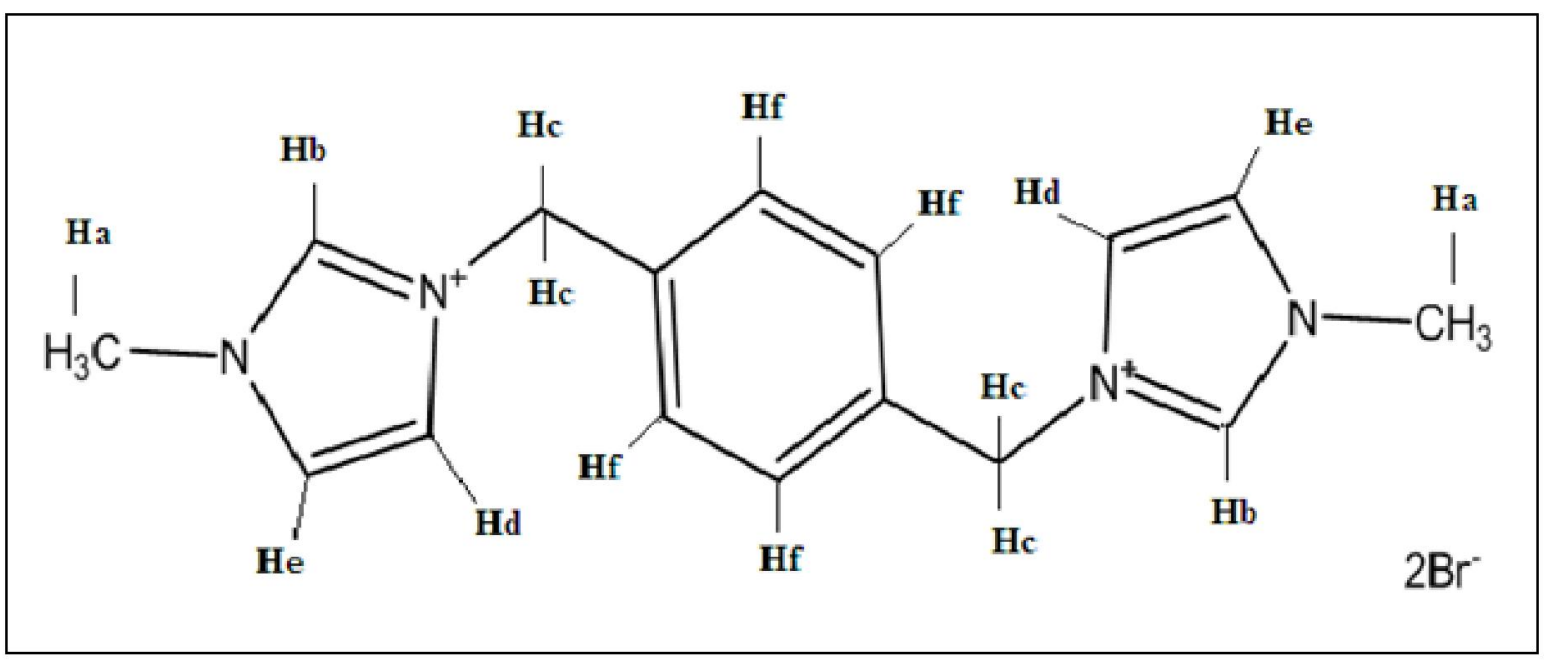




\subsection{Correlation Spectroscopy (COSY)}

To obtain further information of this complex, 2D ${ }^{1} \mathrm{H}-{ }^{1} \mathrm{H}$ COSY spectroscopic technique was studied. 2D NMR is a powerful tool for investigating intermolecular interaction and to gain more information on the conformation of the inclusion complex [33]. The 2D COSY spectrum is essential in determining the interaction between the guest and the host molecules in the complexes and the connectivity between neighboring protons in the inclusion complex [34]. The cross-peaks in the spectra, indicated in Figure 4 originate from the interaction of the protons of PhenmimBr and $\beta-\mathrm{CD}$. The cross peaks of $\beta-\mathrm{CD}$ (3.5-3.6 ppm, H-3, H-5) and PhenmimBr (7.6-7.8 ppm, H-d, e) demonstrates strong intensity. The strong correlation observed suggests that the imidazolium rings of PhenmimBr are strongly interacting with $\beta-C D$. Hence, from the COSY spectra we can conclude that the imidazolium rings of PhenmimBr have entered the cavity of the $\beta-\mathrm{CD}$.

Figure 4. Correlation Spectra of $\beta-C D-P h e n m i m B r$ in DMSO.

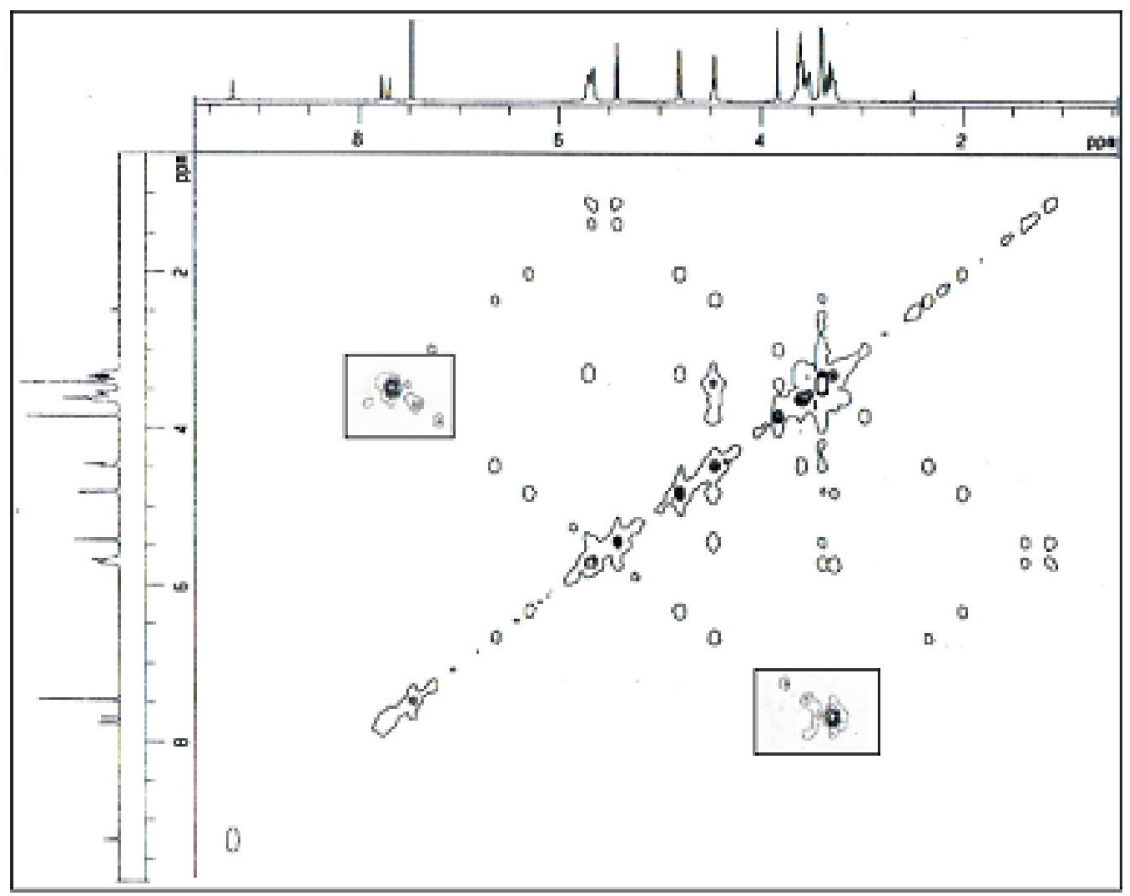

\subsection{Fourier Transform Infrared (FT-IR) Spectroscopy}

FTIR is another useful technique to confirm the formation of an inclusion complex. The FTIR spectrum of $\beta-C D-P h e n m i m B r$ is similar to that of pure $\beta-C D$, which is a major characteristic for the host-guest inclusion complex of $\mathrm{CDs}$ [10]. The most obvious bands: $\mathrm{CH}_{3}(\mathrm{~N})$ stretch, $\mathrm{C}=\mathrm{N}$, imidazolium ring bend $(\mathrm{C}=\mathrm{C})$ and $\mathrm{CH}_{2}$ bend of PhenmimBr were observed in the FTIR spectrum of the $\beta$-CD-PhenmimBr at 2934, 1637, 1580 and $1419 \mathrm{~cm}^{-1}$ respectively (refer to Figure $5 \mathrm{c}$ ). Generally, the intensity and shape of the bands in $\beta$-CD-PhenmimBr were shifted upon inclusion complexation compared to the bands in the free PhenmimBr. The broad $-\mathrm{OH}$ stretching band of the $\beta-\mathrm{CD}$ at $3370 \mathrm{~cm}^{-1}$ corresponds to the multiple $-\mathrm{OH}$ functional groups in $\beta-\mathrm{CD}$ molecules [35] as shown in Figure 5(a). This broad hydroxyl $(-\mathrm{OH})$ band narrows in the spectrum of $\beta-\mathrm{CD}-\mathrm{PhenmimBr}$ (3341 $\left.\mathrm{cm}^{-1}\right)$. Apart from that, $\mathrm{CH}_{2}$ bend of PhenmimBr $\left(1451 \mathrm{~cm}^{-1}\right)$ is reduced and shifts upon 
formation of $\beta$-CD-PhenmimBr $\left(1419 \mathrm{~cm}^{-1}\right)$. In addition $1163 \mathrm{~cm}^{-1}$ absorption peak of $\mathrm{C}-\mathrm{N}$ was also reduced and shifts in $\beta-C D-P h e n m i m B r\left(1155 \mathrm{~cm}^{-1}\right)$. From FT-IR spectra a conclusion can be made, that the formation of inclusion complex between $\beta-\mathrm{CD}$ and PhenmimBr occurs with non-covalent interactions such as hydrophobic interactions, van der Waals interactions and hydrogen bonds which lowers the energy of the included part of PhenmimBr and hence reduces the absorption intensities of the corresponding bonds. The introduction of CD molecules into the IL system occurs with a disruption of the hydrogen bond network which makes ILs potential key tool in the preparation of a new generation of chemical nanostructures [36].

Figure 5. Fourier transform infrared spectra of (a) $\beta-\mathrm{CD}$; (b) PhenmimBr; and (c) $\beta$-CD-PhenmimBr.

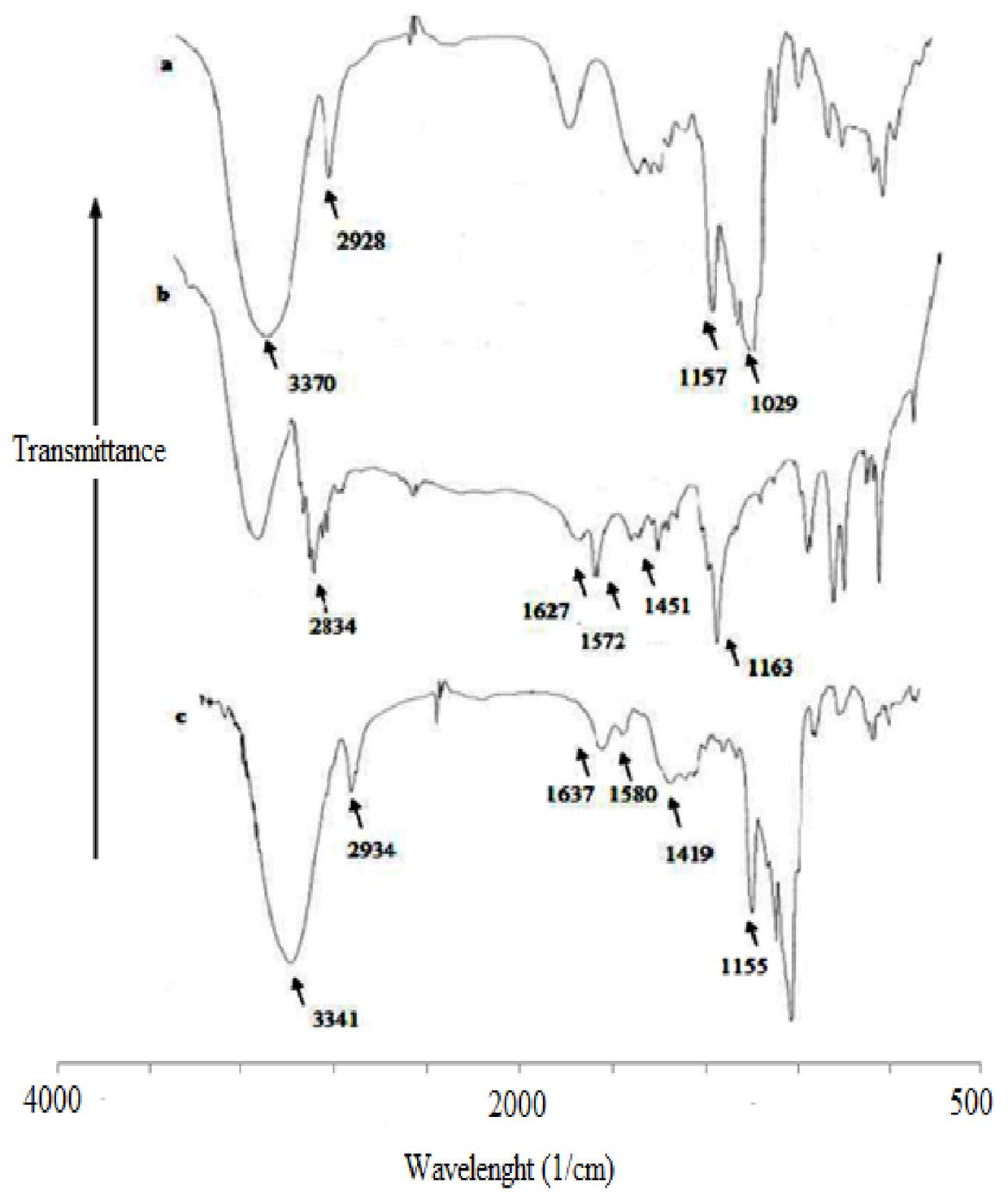




\subsection{X-ray Diffraction (XRD) Analysis}

$\mathrm{X}$-ray diffraction (XRD) provides further evidence for the formation of inclusion structure and it is widely used to determine the formation of a new compound from their parent molecules [35]. Figure 6 shows the XRD patterns of pure $\beta-C D$, PhenmimBr and $\beta-C D-P h e n m i m B r$. Generally, the crystal structures of $\mathrm{CD}$ complexes are categorized mainly into three types: cage-type, channel-type and layer-type. The $\beta$-CD-PhenmimBr obtained was a fine crystalline powder. The results suggested that the XRD pattern of the $\beta$-CD-PhenmimBr was a head-to-head channel type structure [37]. Referring to Figure $6 \mathrm{c}$, the XRD pattern of the $\beta$-CD-PhenmimBr is totally different from the XRD patterns of its parent molecules and this confirms the formation of the inclusion complex. In Figure 6a, major peaks appear at $9.5^{\circ}, 11.1^{\circ}, 12.8^{\circ}, 13.3^{\circ}, 18.8^{\circ}$ and $21.8^{\circ}$ indicating that $\beta$-CD adopted a typical cage structure. While PhenmimBr Figure 6b, shows a typical channel type structure with major peaks appearing at $14.2^{\circ}, 22.3^{\circ}, 24.2^{\circ}$ and $27.5^{\circ}$. Generally, CD molecules are stacked along an axis to form a cylinder for the inclusion complex adopting a head-to-head channel-type structure [10]. The difference between both diffractrograms is due to the interaction between $\beta-\mathrm{CD}$ and PhenmimBr, confirming the formation of the inclusion complex.

Figure 6. X-ray diffractogram of (a) $\beta$-CD; (b) PhenmimBr; and (c) $\beta$-CD-PhenmimBr.

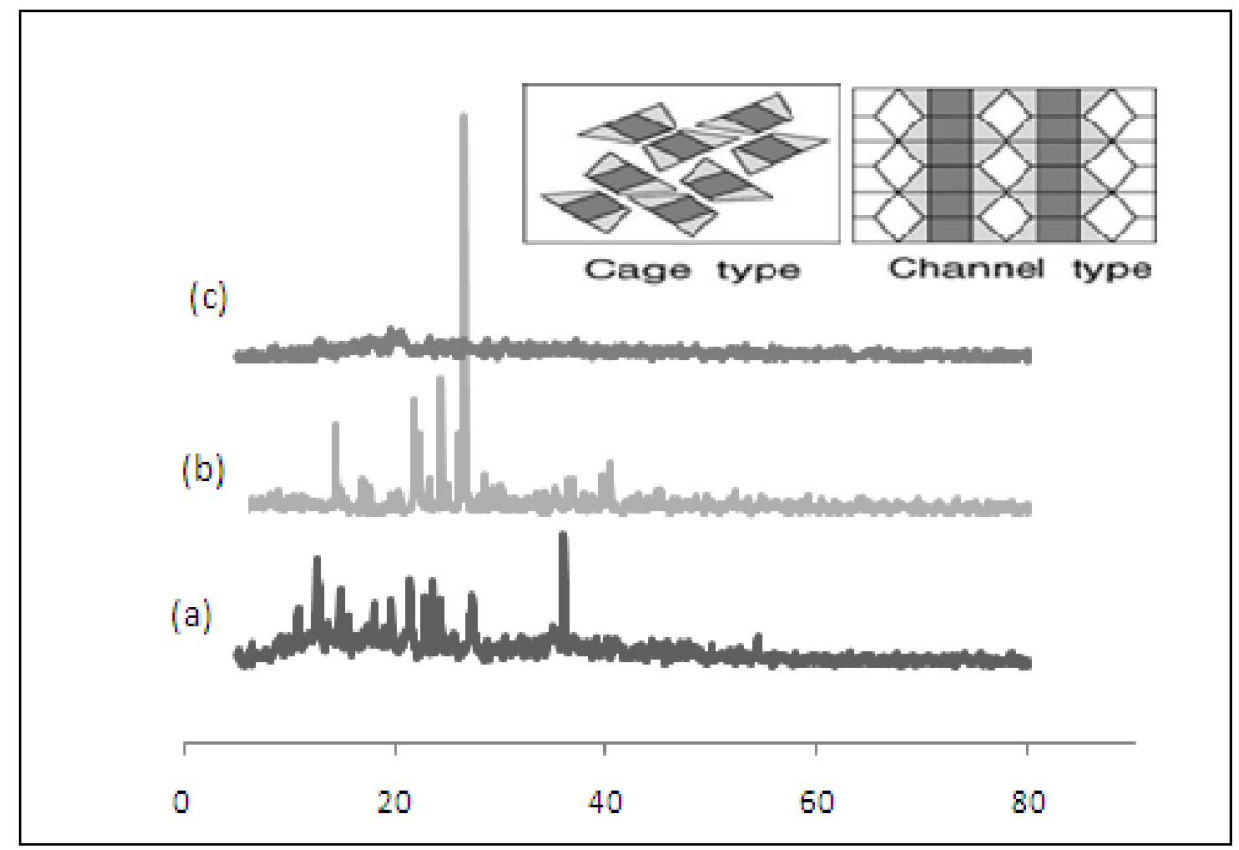

\subsection{Thermogravimetric Analysis (TGA)}

TGA is a frequently used technique to measure a compound's thermal stability. In this study, the thermal stability of $\beta$-CD-PhenmimBr was evaluated using TGA and the results were compared with pure $\beta-C D$ and free PhenmimBr. Figure 7 shows the weight-loss curves for $\beta$-CD-PhenmimBr and its precursors. $\beta$-CD starts to decompose at $320^{\circ} \mathrm{C}$. Visible changes occurred at $120{ }^{\circ} \mathrm{C}$ due to the endothermic behavior which corresponds to loss of water molecules in $\beta$-CD cavity. PhenmimBr starts to decompose at $330{ }^{\circ} \mathrm{C}$. But $\beta$-CD-PhenmimBr starts to decompose at $280{ }^{\circ} \mathrm{C}$, which means the inclusion complex has lowered the initial decomposition temperature of both $\beta-\mathrm{CD}$ and PhenmimBr. 
According to Okumura et al. usually the initial decomposition temperature of CD complexes is higher than those of $\mathrm{CD}$ and the inclusion complexation is supposed to contribute to better stability of CDs [37]. However, the current TGA results are very unusual. This phenomenon may result from the unique molecular structure and properties of the dicationic IL compared to organic compounds and polymers. The steric congestion and the geometry distortion of the components may affect the stability and result in reduction of thermal stability of $\beta-\mathrm{CD}-\mathrm{PhenmimBr}$ [10]. Moreover, when the cation of IL is incorporated within the cavity of $\beta-\mathrm{CD}$, the distance between the cation and anion become larger than in the ion pair, which may lower the decomposition temperature of the complex [25]. Similar phenomenon has been observed in our previous work in which dicationic IL, 1,1',2,2'-tetramethyl-3,3'-p-phenylenedimethylene diimidazolium dibromide (TetraPhimBr) was studied [30].

Figure 7. Thermogravimetric analysis curves of (a) $\beta-\mathrm{CD}$; (b) PhenmimBr; and (c) $\beta$-CD-PhenmimBr.

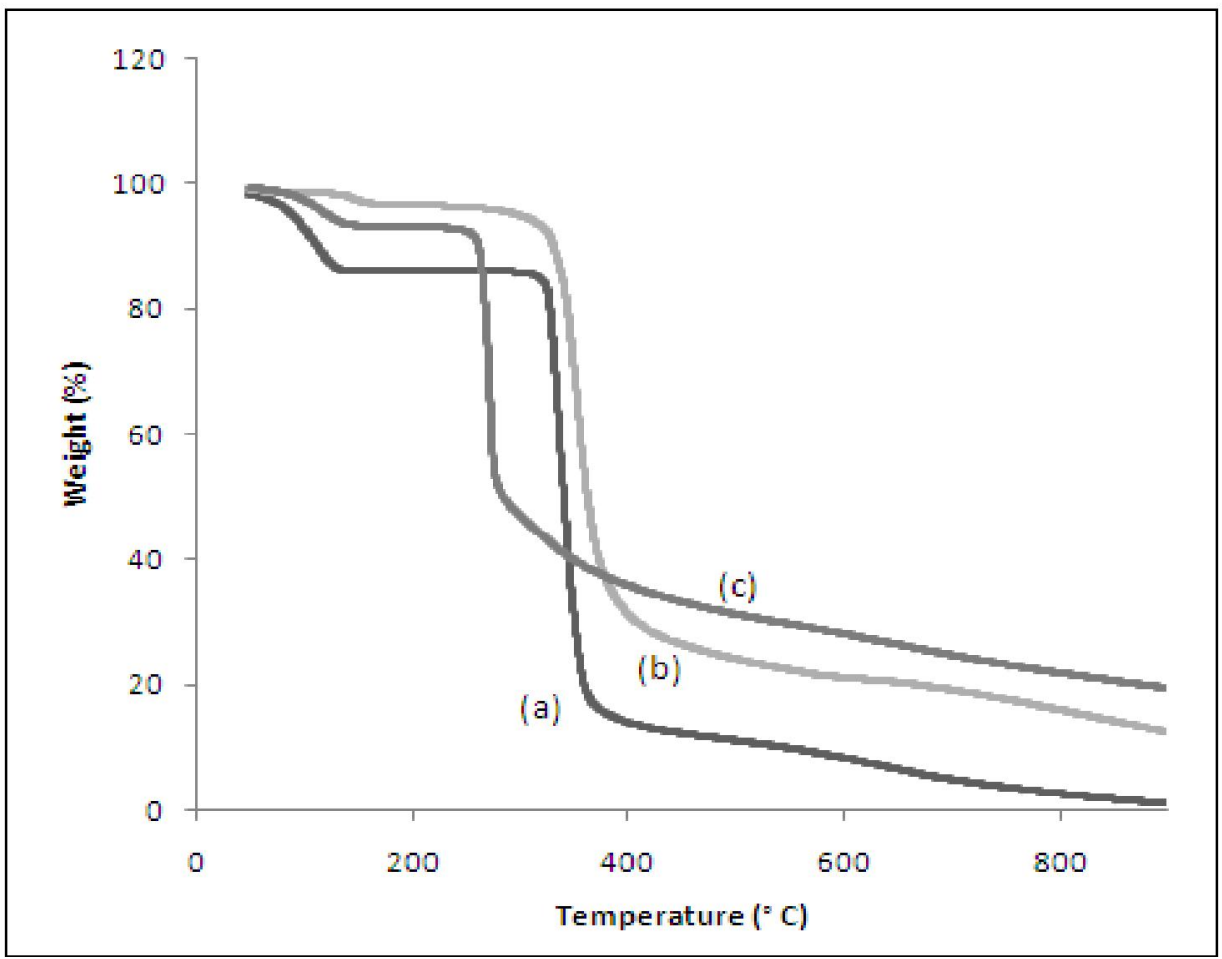

\subsection{Differential Scanning Calorimetry (DSC)}

DSC can be used for the recognition of inclusion complex. When guest molecules are embedded into $\beta-C D$ cavity their melting, boiling or sublimating points generally shifts to different temperatures or disappears. The DSC results are presented in Figure 8 (a) $\beta-C D$, (b) PhenmimBr and (c) $\beta$-CD-PhenmimBr. DSC curve of $\beta$-CD-PhenmimBr is different from its precursor molecules, $\beta-\mathrm{CD}$ and PhenmimBr which further proves the formation of the inclusion complex [38]. As seen from Figure 8a, $\beta$-CD shows two strong endothermic peaks at $70{ }^{\circ} \mathrm{C}$ and $310{ }^{\circ} \mathrm{C}$, the former due to elimination of the high-energy water molecules from $\beta$-CD cavity, the latter due to the decomposition of $\beta$-CD molecule. DSC curve of PhenmimBr exhibited a sharp endothermic peak at $257{ }^{\circ} \mathrm{C}$ which 
corresponds to the melting point of PhenmimBr as shown in Figure $8 \mathrm{~b}$. In the DSC curve of $\beta$-CD-PhenmimBr, the endothermic peaks characteristic of PhenmimBr and $\beta-\mathrm{CD}$ are slightly shifted. Referring to Figure $8 \mathrm{c}$, the peak which is nearly identical to the melting point of pure PhenmimBr has shifted to lower temperature at approximately $247{ }^{\circ} \mathrm{C}$. The other peak corresponding to $\beta$-CD shifts to a higher temperature around $90{ }^{\circ} \mathrm{C}$ which could be explained by the fact when PhenmimBr enters the cavity, the energy of the water molecules in the cavity changes. But surprisingly, a new exothermic peak at $260{ }^{\circ} \mathrm{C}$, which did not exist in any other DSC curve, appears. The presence of this new peak is evidence for formation of the inclusion complex [32].

Figure 8. Differential scanning calorimetry curves of (a) $\beta-\mathrm{CD}$; (b) PhenmimBr; and (c) $\beta$-CD-PhenmimBr.

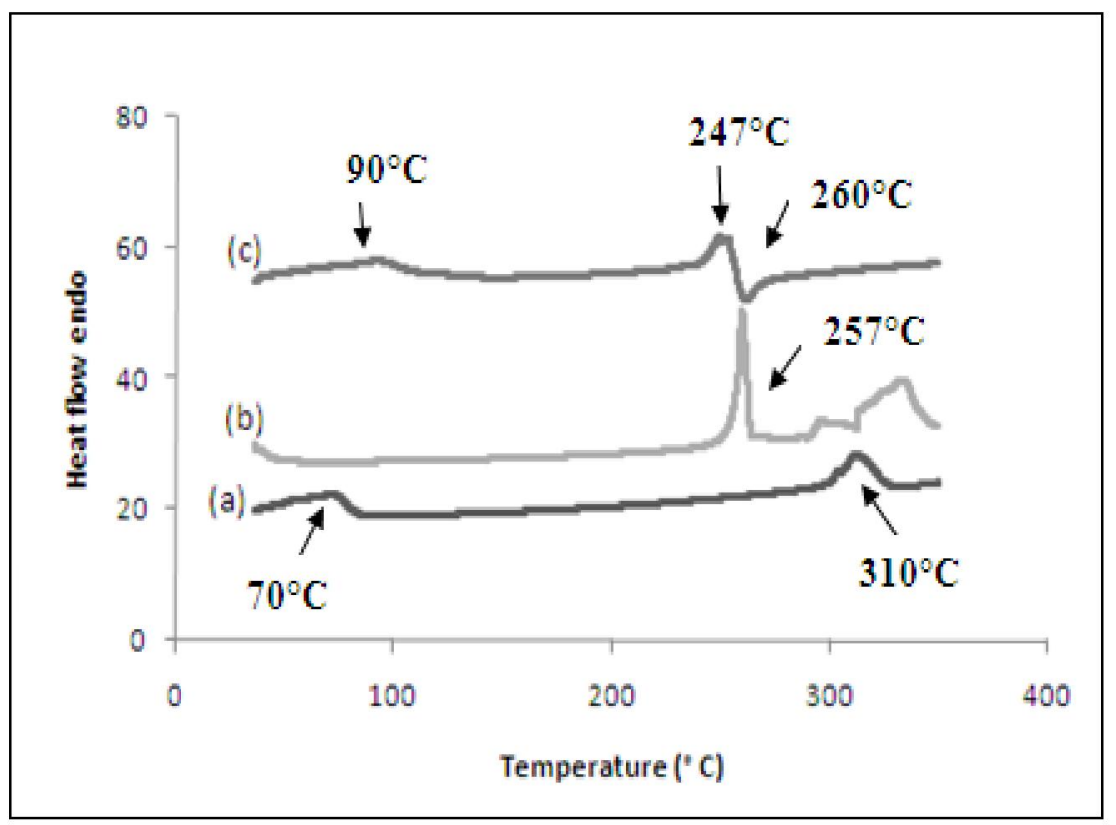

\subsection{Scanning Electron Microscopy (SEM)}

All the microscopic morphological structures were performed using Scanning Electron Microscope (SEM). SEM of $\beta$-CD revealed a "shrinked" crystal structure. It exhibited loss of sphericity, smooth surface and reduced size of particles as shown in Figure 9a. The PhenmimBr appeared as irregularly shaped crystals as shown in Figure 9b. As seen from Figure 9c, $\beta$-CD-PhenmimBr exhibited a totally different crystalline structure, which was not comparable with the morphology of pure $\beta-C D$ and PhenmimBr, thus confirming the formation of $\beta$-CD-PhenmimBr. A drastic change in particle shape and morphology, although not conclusive but it supports the apparent interaction between $\beta-\mathrm{CD}$ and PhenmimBr in solid-state [39]. 
Figure 9. Scanning Electron Microscope photographs of (a) $\beta-C D$; (b) PhenmimBr; and (c) $\beta$-CD-PhenmimBr.

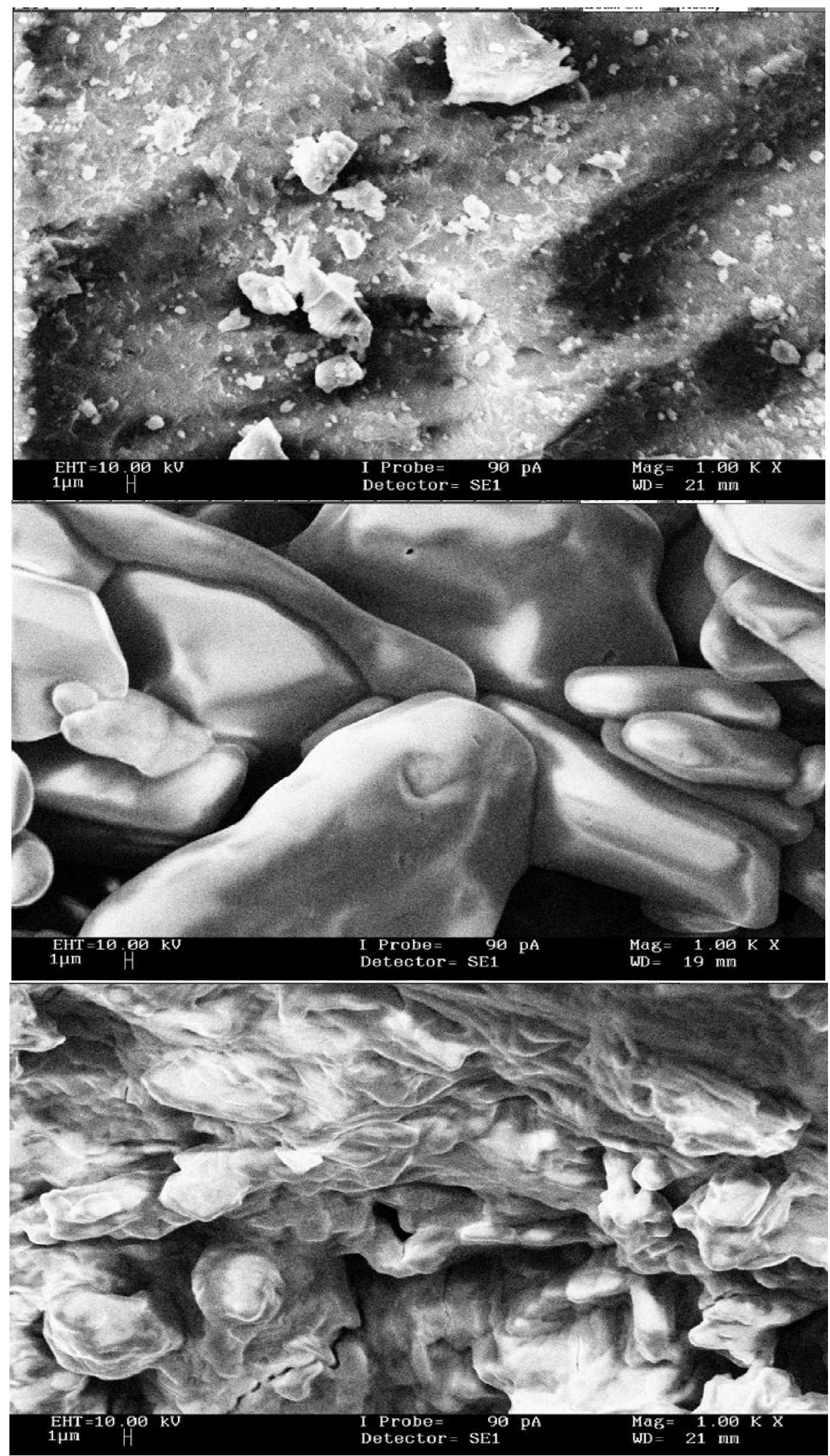




\subsection{UV-Visible Spectroscopy}

The absorption spectra of $\beta$-CD-PhenmimBr complex, PhenmimBr and $\beta$-CD were recorded with accordance to procedure 2.4.1. The obtained result shows that $\beta$-CD had no absorption in the range of 190-250 nm. PhenmimBr was quite similar to $\beta$-CD-PhenmimBr in absorption spectrum shape, but the absorbance of $\beta$-CD-PhenmimBr was higher than that of PhenmimBr at $200 \mathrm{~nm}$ as shown in Figure 10. The influence of the $\beta-\mathrm{CD}$ concentration on PhenmimBr was also studied. As can be seen from Figure 11, addition of $\beta-C D$ had caused a noticeable increase in the absorption intensity. This is because upon inclusion in the $\beta$-CD cavity, generally the absorbance of the guest molecule will be enhanced due to shielding of the excited species from non-radiative processes occurring in the bulk solution and also due to increase in molar absorption coefficient of the inclusion complex ( $\beta$-CD-PhenmimBr).

Figure 10. Absorption spectra of (a) $\beta-\mathrm{CD}$; (b) PhenmimBr; and (c) $\beta$-CD-PhenmimBr with [PhenmimBr]: $0.01 \mathrm{mM}$ and $[\beta-\mathrm{CD}]: 0.0032 \mathrm{M}$; at $\mathrm{pH} 7, T=25^{\circ} \mathrm{C}$.

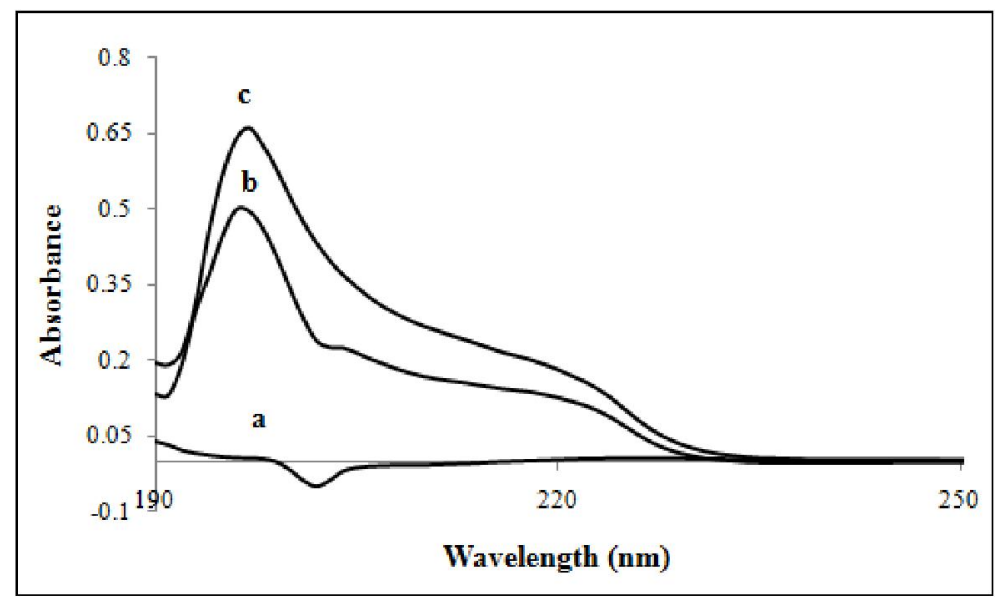

Figure 11. Absorption spectra of PhenmimBr with various concentration of $\beta-\mathrm{CD}$ at $\mathrm{pH} 7$, $T=25^{\circ} \mathrm{C}$. From lines 1 to $5: 0 \mathrm{M} ; 0.001 \mathrm{M} ; 0.002 \mathrm{M} ; 0.003 \mathrm{M}$ and $0.005 \mathrm{M}$.

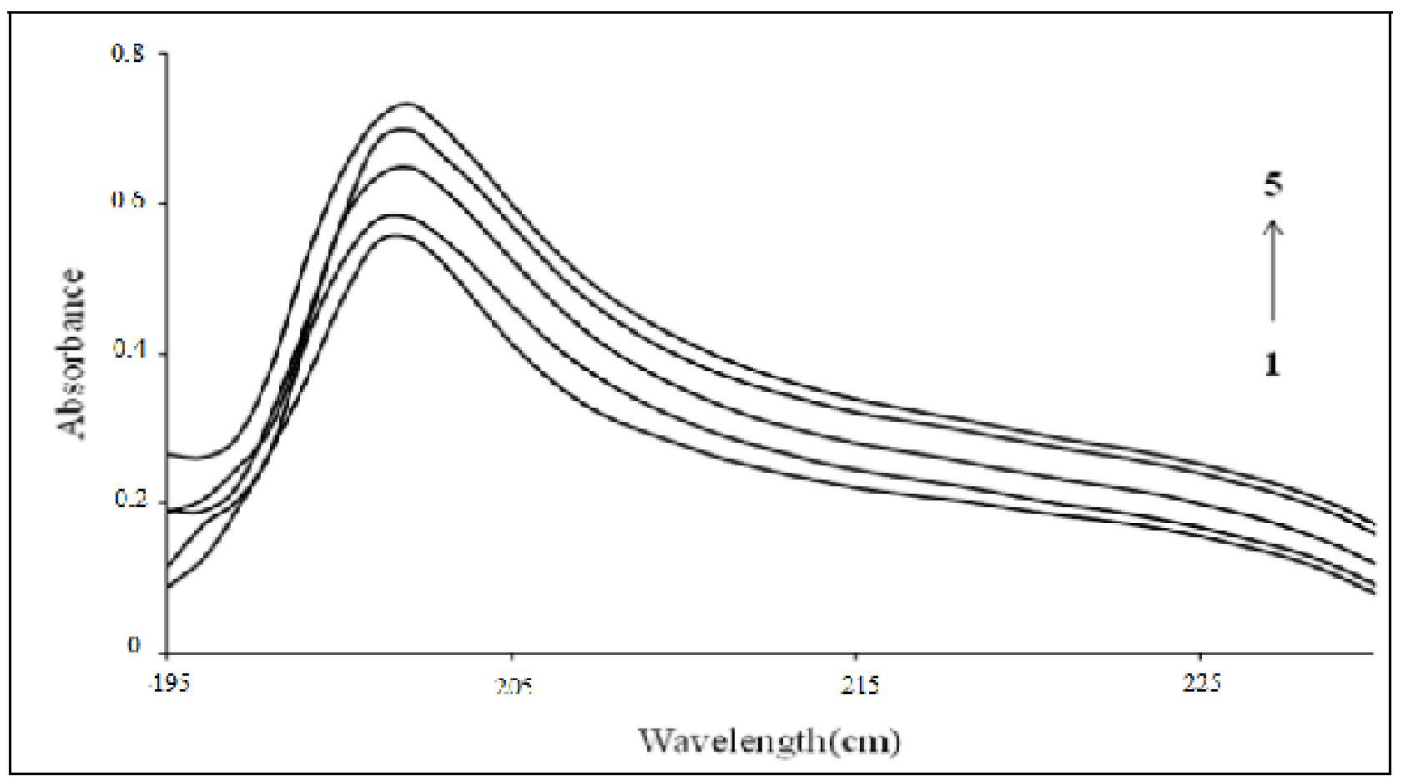




\subsubsection{Stoichiometry of the Complex and Formation Constant}

The formation constant is the most important parameter in the study of inclusion behavior of $\beta$-CD. The formation constant for the inclusion complex has been determined by analyzing the changes in the intensities of absorption with different $\beta-C D$ concentrations. The formation constant is denoted by $\mathrm{K}$ and the stoichiometric ratio of the $\beta$-CDPhenmimBr was determined using the modified Benesi-Hildebrand equation [40]. The $\mathrm{K}$ value was calculated by dividing the slope with the intercept of straight line obtained from the double reciprocal plot. A good linear relationship was obtained when 1/Absorbance is plotted against $1 / \beta-\mathrm{CD}^{2}$ with $R^{2}=0.9936$ for $\beta$-CD-PhenmimBr (Figure 12), indicating that the stoichiometric ratio of the complex formed is $2: 1$. The apparent formation constant is determined to be $2.61 \times 10^{5} \mathrm{~mol}^{-2} \mathrm{~L}^{2}$. The dicationic IL (PhenmimBr) have higher formation constant value compared to monocationic ILs with reference to the tabulated results obtained by He et al. [28]. The formation constant for $\mathrm{BmimCl}$ and $\mathrm{OmimBr}$ is 8.16 and $672 \mathrm{~L} / \mathrm{mol}$ respectively [28] which is lower compared to the value obtained for PhenmimBr. It is quite conclusive that dicationic IL have a greater tendency to bind strongly with $\beta$-CD than monocationic ILs. Furthermore, we suggest that the dicationic IL tends to form inclusion complex in 2:1 ratio due to the presence of two imidazolium rings meanwhile monocationic ILs have greater tendency to form 1:1 inclusion complex.

Figure 12. Reciprocal plot of $1 /$ Absorbance versus $1 /[\beta-C D]^{2}$.

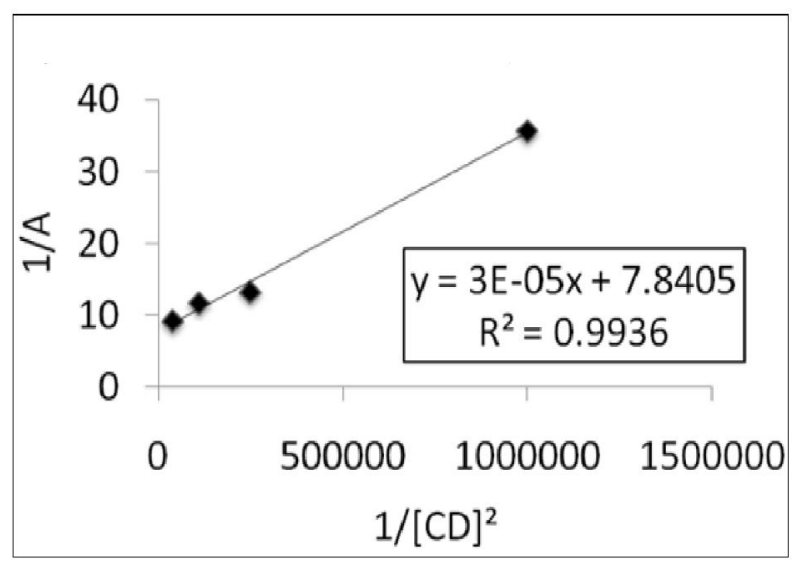

\subsubsection{Inclusion Structure}

The possible inclusion mechanism is proposed from the stoichiometric ratio (2:1) obtained. Furthermore, based on the ${ }^{1} \mathrm{H}$ NMR and COSY results, the proposed structure of $\beta$-CD-PhenmimBr is presented in Figure 13. Based on our findings, it is very obvious that the imidazolium ring has entered the cavity of $\beta-C D$. But in contrast, He et al. recently had claimed that only the alkyl side chain of the IL interacts with $\beta$-CD while the imidazolium ring does not interact [28]. This discrepancy can be explained by the fact that the hydrophobic interaction and relatively larger size of the dicationic IL, PhenmimBr plays an important role in the inclusion process. The cation without long hydrocarbon chain can be included into the cavity of $\beta$-CD. However the key problem is the location of anion. It has been discovered that perhaps, anion dissociates near $\beta-\mathrm{CD}$ molecules. 
Figure 13. Proposed structure for possible inclusion mechanism.

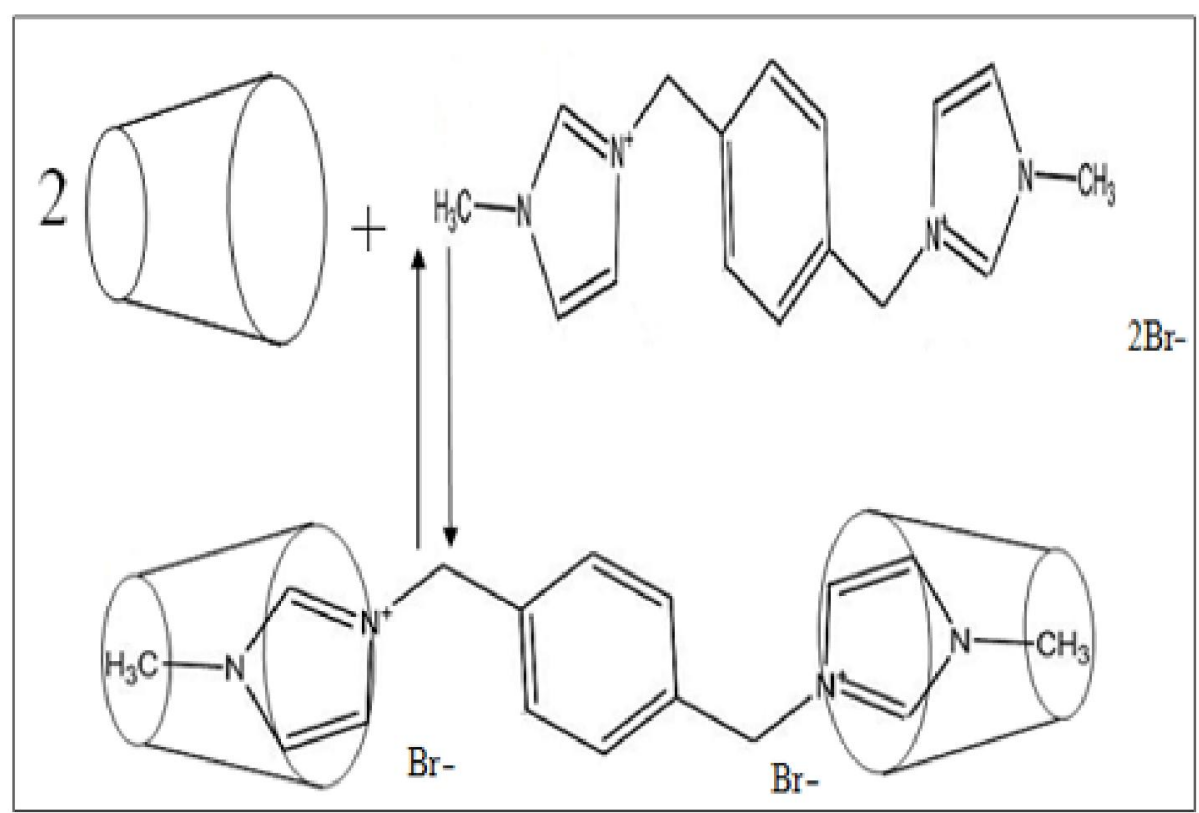

\section{Conclusion}

The inclusion behavior of $\beta$-CD with dicationic IL, PhenmimBr has been investigated. The results obtained by different characterization techniques clearly indicates that the kneading method leads to the formation of a new product ( $\beta$-CD-PhenmimBr), with properties different from those of originating host $(\beta-C D)$ and guest molecules (PhenmimBr). Based on the FT-IR, ${ }^{1} \mathrm{H}$ NMR and COSY spectra, it is found that the imidazolium ring of PhenmimBr has entered the hydrophobic cavity of $\beta$-CD. The dicationic IL (PhenmimBr) exhibits higher formation constant value $\left(2.61 \times 10^{5} \mathrm{~mol}^{-2} \mathrm{~L}^{2}\right)$ compared to monocationic ILs. This suggests that the imidazolium rings in dicationic IL binds strongly with the hydrophobic cavity of $\beta-C D$ which enables it to be applied in separation studies. Currently we are investigating the possibility of this complex as adsorbent for solid phase extraction.

\section{Acknowledgement}

This work was financially supported by, University of Malaya Centre for Ionic Liquids (grant no. TA010-2010A) and University Research Grant Scheme (RG055/09SUS).

\section{Reference}

1. Szejtli, J. Introduction and general overview of cyclodextrin chemistry. Chem. Rev. 1998, 98, 1743-1753.

2. Ashnagar, A.; Naseri, N.G.; Khanaki, B. Study of the $\beta$-cyclodextrin imipramine hydrochloride inclusion complex and determination of its stability constant $(\mathrm{K})$ by UV-Visible spectroscopy. E-J. Chem. 2007, 4, 550-558.

3. Letellier, S.; Maupas, B.; Gramond, J.P.; Guyon, F.; Gareil, P. Determination of the formation constant for the inclusion complex between rutin and methyl- $\beta$-cyclodextrin. Anal. Chim. Acta 1995, 315, 357-363. 
4. Schneiderman, E.; Stalcup, A.M. Cyclodextrins: A versatile tool in separation science. J. Chromatogr. B 2000, 745, 83-102.

5. Bernini, A.; Spiga, O.; Ciutti, A.; Scarselli, M.; Bottoni, G.; Mascagni, P.; Niccolai, N. NMR studies of the inclusion complex between beta-cyclodextrin and paroxetine. Eur. J. Pharm. Sci. 2004, 22, 445-450.

6. Bonenfant, D.; Niquette, P.; Mimeault, M.; Furtos-Matei, A.; Hauslera, R. UV-VIS and FTIR spectroscopic analyses of inclusion complexes of nonylphenol and nonylphenol ethoxylate with $\beta$-cyclodextrin. Water Res. 2009, 43, 3575-3581.

7. Waleczek, K.J.; Marques, C.; Hempel, B.; Schmidt, P.C. Phase solubility study of pure (-)- $\alpha$-bisabolol and camomile essential oil with $\beta$-cyclodextrin. Eur. J. Pharm. Biopharm. 2003, 55, 247-251.

8. He, Y.; Shen, X. Interaction between $\beta$-cyclodextrin and ionic liquids in aqueous solutions investigated by a competitive method using a substituted $3 \mathrm{H}$-indole probe. J. Photochem. Photobiol. Chem. 2008, 197, 253-259.

9. Yáñez, C.; Salazar, R.; Núñez-Vergara, L.J.; Squella, J.A. Spectrophotometric and electrochemical study of the inclusion complex between $\beta$-cyclodextrin and furnidipine. J. Pharm. Biomed. Anal. 2004, 35, 51-56.

10. Li, N.; Zhao, X.; Gao, Y.; Zheng, L.; Zhang, J.; Yu, L. Complex formation of ionic liquid surfactant and $\beta$-cyclodextrin. Colloids Surf. A 2007, 292, 196-201.

11. Tavornvipas, S.; Hirayama, F.; Arima, H.; Uekama, K.; Ishiguro, T.; Oka, M.; Hamayasu, K.; Hashimoto, H. 6-O-a-(4-O-a-D-glucuronyl)-D-glucosyl- $\beta$-cyclodextrin solubilizing ability and some cellular effects. Int. J. Pharm. 2002, 249, 199-209.

12. Padukka, I.; Bhandari, B.; Darcy, B. Evaluation of various extraction methods of encapsulated oil from $\beta$-cyclodextrin-lemon oil complex powder. J. Food Compos. Anal. 2000, 13, 59-70.

13. Szente, L.; Szejtli, J. Cyclodextrins as food ingredients. Trends Food Sci. Technol. 2004, 15, 137-142.

14. Perrin, C.; Fabre, H.; Maftouh, M.; Massart, D.L.; Heyden, Y.V. Robustness testing of chiral separations by capillary electrophoresis using highly-sulfated cyclodextrins. J. Chromatogr. A 2003, 1007, 165-177.

15. Morillo, E.; Perez-Martinez, J.I.; Gines, J.M. Leaching of 2,4-D from a soil in the presence of $\beta$-cyclodextrin: Laboratory columns experiments. Chemosphere 2001, 44, 1065-1069.

16. Jozefaciuk, G.; Muranyi, A.; Fenyvesi, E. Effect of randomly methylated $\beta$-cyclodextrin on physical properties of soils. Environ. Sci. Technol. 2003, 37, 3012-3017.

17. McEwen, A.B.; Ngo, H.L.; LeCompte, K.; Goldman, J.L. Electrochemical properties of imidazolium salt electrolytes for electrochemical capacitor applications. J. Electrochem. Soc. 1999, 146, 1687-1695.

18. Xue. H.; Shreeve, M.J. Ionic Liquids with Fluorine-Containing Cations. Eur. J. Inorg. Chem. 2005, 13, 2573-2580.

19. Wilkes, J.S. Ionic Liquid in Synthesis; Wasserscheid, P., Welton, T., Eds.; Wiley-VCH: Hoboken, NJ, USA, 2002.

20. Anderson, J.L.; Ding, R.; Ellern, A.; Armstrong, D.W. Structure and properties of high stability geminal dicationic ionic liquids. J. Am. Chem. Soc. 2005, 127, 593-604. 
21. Qi, S.; Cui, S.; Chen, X.; Hu, Z. Rapid and sensitive determination of anthraquinones in Chinese herb using 1-butyl-3-methylimiazolium-based ionic liquid with $\beta$-cyclodextrin as modifier in capillary zone electrophoresis. J. Chromatogr. A 2004, 1059, 191-198.

22. Yu, L.; Qin, W.; Li, S.F.Y. Ionic liquids as additives for separation of benzoic acid and chlorophenoxy acid herbicides by capillary electrophoresis. Anal. Chim. Acta 2006, 547, 165-171.

23. Jing, B.; Chen, X.; Hao, J.C.; Qiu, H.Y.; Chai, Y.C.; Zhang, G.D. Supramolecular self-assembly of polypseudorotaxanes in ionic liquid. Colloids Surf. A 2007, 292, 51-55.

24. Tian, K.; Wang, Y.S.; Chen, Y.L.; Chen, Y.G.; Hu, Z.D. Application of 1-alkyl-3methylimidazolium-based ionic liquids as background electrolyte in capillary zone electrophoresis for the simultaneous determination of five anthraquinones in Rhubarb. Talanta 2007, 72, 587-593.

25. Gao, Y.A.; Li, Z.H.; Du, J.M.; Han, B.X.; Li, G.Z.; Hou, W.G.; Shen, D.; Zheng, L.Q.; Zhang, G.Y. Preparation and characterization of inclusion complexes of $\beta$-cyclodextrin with ionic liquid. Chem. Eur. J. 2005, 11, 5875-5880.

26. Gao, Y.; Zhao, X.; Dong, B.; Zheng, L.; Li, N.; Zhang, S. Inclusion complexes of $\beta$-cyclodextrin with ionic liquid surfactants. J. Phys. Chem. B 2006, 110, 8576-8581.

27. Amajjahe, S.; Ritter, H. Anion complexation of vinylimidazolium salts and its influence on polymerization. Macromolecules 2008, 41, 716-718.

28. He, Y.; Chen, Q.; Xu, C.; Zhang, J.; Shen, X. Interaction between ionic liquid and $\beta$ Cyclodextrin: A discussion of association pattern. J. Phys. Chem. B 2009, 113, 231-238.

29. Li, H.G.; Zhang, Q.; Zhao, Q.; Liu, M.; Liu, J.; Sun, D.Z. Studies on interaction of ionic liquids with cyclodextrin aqueous solution. Indian J. Chem. 2010, 49A, 752-756.

30. Puvaneswary, S.; Sharifah, M.; Alias, Y. Synthesis and characterization of the inclusion complex of dicationic ionic liquid and $\beta$-cyclodextrin. Int. J. Mol. Sci. 2010, 11, 3675-3685.

31. Kilivelu, G.; Yatimah, A. Synthesis and characterization of novel dimeric ionic liquids by conventional approaches. Int. J. Mol. Sci. 2008, 9, 1207-1213.

32. Cwiertnia, B.; Hladon, T.; Stobiecki, M. Stability of diclofenac sodium in the inclusion complex with $\beta$-cyclodextrin in the solid state. J. Pharm. Pharmacol. 1999, 51, 1213-1218.

33. Li, J.; Ni, X.; Zhou, Z.; Leong, K.W. Preparation and characterization of polypseudorotaxanes based on block-selected inclusion complexation between poly(propylene oxide)-poly(ethylene oxide)-poly(propylene oxide) triblock copolymers and $\alpha$-cyclodextrin. J. Am. Chem. Soc. 2003, 125, 1788-1795.

34. Qian, L.; Guan, Y.; Xiao, H. Preparation and characterization of inclusion complexes of a cationic $\beta$-cyclodextrin polymer with butylparaben or triclosan. Int. J. Pharm. 2008, 357, 244-251.

35. Nostro, P.L.; Santoni, I.; Bonini, M.; Baglioni, P. Inclusion compound from a semifluorinated alkane and $\beta$-cyclodextrin. Langmuir 2003, 19, 2313-2317.

36. Dupont, J. On the solid, liquid and solution structural organization of imidazolium ionic liquids. J. Braz. Chem. Soc. 2004, 15, 341-350.

37. Okumura, H.; Kawaguchi, Y.; Harada, A. Preparation and characterization of the inclusion complexes of poly(dimethylsilane)s with cyclodextrins. Macromolecules 2003, 36, 6422-6429.

38. Liu, L.; Zhu, S. A study on the supramolecular structure of inclusion complex of $\beta$-cyclodextrin with prazosin hydrochloride. Carbohydr. Polym. 2007, 68, 472-476. 
39. Pralhad, T.; Rajendrakumar, K. Study of freeze-dried quercetin-cyclodextrin binary systems by DSC, FT-IR, X-ray diffraction and SEM analysis. J. Pharmaceut. Biomed. Anal. 2004, 34, 333-339.

40. Wang, H.Y.; Han, J.; Feng, X.G. Spectroscopic study of orange G- $\beta$-cyclodextrin complex and its analytical application. Spectrochim. Acta Part A 2007, 66, 578-585.

(C) 2011 by the authors; licensee MDPI, Basel, Switzerland. This article is an open access article distributed under the terms and conditions of the Creative Commons Attribution license (http://creativecommons.org/licenses/by/3.0/). 